Cyclotomic and simplicial matroids (with Victor Reiner) Israel J. Math. 150 (2005), 229--240. The original publication is available at www.springerlink.com: http://dx.doi.org/10.1007/BF02762381, Open Access version: http://kuscholarworks.ku.edu/dspace/.

[This document contains the author's accepted manuscript. For the publisher's version, see the link in the header of this document.]

\title{
CYCLOTOMIC AND SIMPLICIAL MATROIDS
}

\author{
JEREMY L. MARTIN AND VICTOR REINER
}

\begin{abstract}
We show that two naturally occurring matroids representable over $\mathbb{Q}$ are equal: the cyclotomic matroid $\mu_{n}$ represented by the $n^{\text {th }}$ roots of unity $1, \zeta, \zeta^{2}, \ldots, \zeta^{n-1}$ inside the cyclotomic extension $\mathbb{Q}(\zeta)$, and a direct sum of copies of a certain simplicial matroid, considered originally by Bolker in the context of transportation polytopes. A result of Adin leads to an upper bound for the number of $\mathbb{Q}$-bases for $\mathbb{Q}(\zeta)$ among the $n^{\text {th }}$ roots of unity, which is tight if and only if $n$ has at most two odd prime factors. In addition, we study the Tutte polynomial of $\mu_{n}$ in the case that $n$ has two prime factors.
\end{abstract}

\section{INTRODUCTION}

This paper is about two matroids representable over $\mathbb{Q}$ that turn out, somewhat unexpectedly, to be dual (or orthogonal). Briefly, a matroid is a combinatorial abstraction of the linear dependence data associated to a (finite) set of vectors in a vector space. That is, the data for a matroid on ground set $E$ records which subsets of $E$ are dependent and independent, or the linear span and dimension of each subset, etc. The matroid is representable over a field $\mathbb{F}$ if the elements of $E$ may be identified with vectors in an $\mathbb{F}$-vector space that achieve the matroid data. The dual of a matroid $M$ on $E$ is defined generally as the matroid $M^{*}$ whose bases are the complements of bases in $M$; this abstracts the situation where $M, M^{*}$ are matroids represented over $\mathbb{F}$ by the columns of two matrices whose row spaces are orthogonal complements of each other. For background, motivation, and matroid terminology left undefined here, see any of the standard references $[6,13,15,16,17,18]$.

The first matroid that we study relates to cyclotomic extensions. Let $\zeta_{n}$ be a primitive $n^{\text {th }}$ root of unity, which we will abbreviate by $\zeta$ when no confusion can arise. Recall that the degree of the cyclotomic extension $\mathbb{Q}(\zeta)$ is given by Euler's $\phi$-function $\phi(n)$, with the following formula: if $n=p_{1}^{m_{1}} \cdots p_{r}^{m_{r}}$ for distinct primes $p_{i}$, then

$$
\phi(n)=p_{1}^{m_{1}-1} \cdots p_{r}^{m_{r}-1}\left(p_{1}-1\right) \cdots\left(p_{r}-1\right) .
$$

2000 Mathematics Subject Classification. Primary 05B35; Secondary 11R18,55U10.

Key words and phrases. Cyclotomic extension, simplicial matroid, higher-dimensional tree, transportation polytope, Tutte polynomial.

First author supported by NSF Postdoctoral Fellowship. Second author supported by NSF grant DMS-0245379. 
[This document contains the author's accepted manuscript. For the publisher's version, see the link in the header of this document.]

The $\mathbb{Q}$-vector space $\mathbb{Q}(\zeta)$ gives rise to a natural matroid $\mu_{n}$, represented by the $n$ vectors $Z_{n}=\left\{1, \zeta, \zeta^{2}, \ldots, \zeta^{n-1}\right\}$ and having rank $\phi(n)$. We call $\mu_{n}$ the cyclotomic matroid of order $n$.

The second matroid is a simplicial matroid [17, Chapter 6]; that is, it is represented by the columns of a simplicial boundary map (see, e.g., [12] for background on simplicial homology). Let $\Delta=\Delta^{d}$ be a $d$-dimensional simplicial complex $^{1}$ whose (reduced) simplicial homology with coefficients in $\mathbb{F}$ vanishes in codimension 1 ; that is, $\tilde{H}_{d-1}(\Delta, \mathbb{F})=0$. If $\Delta$ has exactly $n$ facets (that is, faces of maximum dimension $d$ ), then the $n$ columns in the matrix expressing the simplicial boundary map

$$
\tilde{C}_{d}\left(\Delta^{d}, \mathbb{F}\right) \stackrel{\partial_{d}}{\longrightarrow} \tilde{C}_{d-1}\left(\Delta^{d}, \mathbb{F}\right)
$$

represent a matroid over $\mathbb{F}$ that we will call the simplicial matroid $\mathcal{S}\left(\Delta^{d}, \mathbb{F}\right)$. This is a matroid on $n$ elements, with

$$
\operatorname{rank} \mathcal{S}\left(\Delta^{d}, \mathbb{F}\right)=\operatorname{dim}_{\mathbb{F}} \operatorname{im} \partial_{d}=\operatorname{dim}_{\mathbb{F}} \operatorname{ker} \partial_{d-1} .
$$

In this paper, we consider the $(r-1)$-dimensional simplicial complex $\Delta_{n_{1}, \ldots, n_{r}}^{r-1}$ defined as the simplicial join $[12, \S 62]$ of 0 -dimensional complexes $\Delta_{n_{1}}^{0}, \ldots, \Delta_{n_{r}}^{0}$, where $\Delta_{n_{i}}^{0}$ consists of $n_{i}$ disjoint vertices. That is, a face of $\Delta_{n_{1}, \ldots, n_{r}}^{r-1}$ contains at most one vertex from each $\Delta_{n_{i}}^{0}$. Our main result expresses the connection between cyclotomic matroids and these particular simplicial matroids.

Theorem 1. Let $n=p_{1}^{m_{1}} \cdots p_{r}^{m_{r}}$, with $p_{1}, \ldots, p_{r}$ distinct primes and $m_{1}, \ldots, m_{r}$ positive integers.

Then the following two matroids representable over $\mathbb{Q}$ are dual:

- The cyclotomic matroid $\mu_{n}$.

- The direct sum of $p_{1}^{m_{1}-1} \cdots p_{r}^{m_{r}-1}$ copies of $\mathcal{S}\left(\Delta_{p_{1}, \ldots, p_{r}}^{r-1}, \mathbb{Q}\right)$.

Bolker [5] was the first to study the simplicial matroid $\mathcal{S}\left(\Delta_{n_{1}, \ldots, n_{r}}^{r-1}, \mathbb{Q}\right)$, where the $n_{i}$ are positive integers, not necessarily prime. He proposed the bases of this matroid as higher-dimensional analogues of spanning trees ${ }^{2}$ in bipartite graphs, and studied their relation to vertices of certain transportation polytopes. Each such basis $T$ of $\mathcal{S}\left(\Delta_{n_{1}, \ldots, n_{r}}^{r-1}, \mathbb{Q}\right)$ gives rise to a $\mathbb{Q}$-acyclic pure $(r-1)$-dimensional simplicial complex $\Delta_{T}$, obtained by attaching the $(r-1)$-simplices indexed by $T$ to the $(r-2)$-skeleton of $\Delta_{n_{1}, \ldots, n_{r}}^{r-1}$. He also suggested

$$
\prod_{i=1}^{r} n_{i}^{\prod_{j \neq i}\left(n_{j}-1\right)}
$$

\footnotetext{
${ }^{1}$ We adopt the following notational convention throughout. When we wish to emphasize that a simplicial complex $\Delta$ has dimension $d$, we will denote it by the symbol $\Delta^{d}$; otherwise, we will frequently omit the superscript to simplify notation.

${ }^{2}$ This is not the only way to generalize the notion of spanning tree to 2- or higher-dimensional simplicial complexes; see, e.g., [4], [7], [8]. These other generalizations, however, play no role in our present study.
} 
Cyclotomic and simplicial matroids (with Victor Reiner) Israel J. Math. 150 (2005), 229--240. The original publication is available at www.springerlink.com: http://dx.doi.org/10.1007/BF02762381, Open Access version: http://kuscholarworks.ku.edu/dspace/.

[This document contains the author's accepted manuscript. For the publisher's version, see the link in the header of this document.]

as an upper bound for the number of bases, and showed that this formula gives the exact number of bases if and only if at most two of the $n_{i}$ exceed 2 . In particular, Bolker showed [5, Theorems 27, 28] that this occurs exactly when for each basis $T$, the (pure torsion) integer homology group $\tilde{H}_{r-2}\left(\Delta_{T}, \mathbb{Z}\right)$ is trivial.

A result of Adin [3] (generalizing work of Kalai in [10]) implies that (1) is indeed an upper bound for the number of bases of $\Delta_{n_{1}, \ldots, n_{r}}^{r-1}$. The method of Adin and Kalai is to generalize the classical Kirchhoff Matrix-Tree Theorem to simplicial complexes, using the Binet-Cauchy determinant formula: this yields the result

$$
\sum_{\substack{\text { bases } T \text { of } \\ \mathcal{S}\left(\Delta_{n_{1}, \ldots, n_{r}}^{r-1}, \mathbb{Q}\right)}}\left|\tilde{H}_{r-2}\left(\Delta_{T}, \mathbb{Z}\right)\right|^{2}=\prod_{i=1}^{r} n_{i}^{\prod_{j \neq i}\left(n_{j}-1\right)} .
$$

That is, Bolker's formula (1) is an exact count for such bases with the following weighting: instead of a basis $T$ contributing 1 to the count, its contribution is the square of the order of its integral homology group in codimension 1.

Combining the results of Adin and Bolker with Theorem 1, and the fact that dual matroids have the same number of bases, one immediately obtains the following corollary.

Corollary 2. Let $n=p_{1}^{m_{1}} \cdots p_{r}^{m_{r}}$, with $p_{1}, \ldots, p_{r}$ distinct primes and $m_{1}, \ldots, m_{r}$ positive integers, and let $\zeta$ be a primitive $n^{\text {th }}$ root of unity.

Then the number of subsets of $Z_{n}=\left\{1, \zeta, \zeta^{2}, \ldots, \zeta^{n-1}\right\}$ that are bases for $\mathbb{Q}(\zeta)$ is bounded above by

$$
\left(\prod_{i=1}^{r} p_{i} \prod_{j \neq i}\left(p_{j}-1\right)\right)^{p_{1}^{m_{1}-1} \cdots p_{r}^{m_{r}-1}}
$$

with equality if and only if $n=2^{a} p^{b} q^{c}$ for nonnegative integers $a, b, c$.

Section 2 contains the proof of Theorem 1. In Section 3, we study the case that $n=p_{1}^{m_{1}} p_{2}^{m_{2}}$ has only two prime factors. Here the simplicial complex $\Delta_{p_{1}, p_{2}}^{1}$ is just the complete bipartite graph $K_{p_{1}, p_{2}}$, and the matroid $\mathcal{S}\left(\Delta_{p_{1}, p_{2}}^{1}, \mathbb{Q}\right)$ is the cycle matroid (or graphic matroid) of $K_{p_{1}, p_{2}}$, whose bases are spanning trees. We study enumerative invariants finer than the number of bases for these particular graphic matroids, such as their Tutte polynomials, and a weighted enumeration of their spanning forests.

\section{Proof of Theorem 1}

We first state a well-known general fact about matroids, to be used in the proof.

Lemma 3. Let $M$ be a matroid of rank $\rho$ on ground set $E$. Suppose there exists $a$ (disjoint) decomposition $E=\bigsqcup_{i=1}^{t} E_{i}$ such that $\operatorname{rank}(M)=\sum_{i=1}^{t} \operatorname{rank}\left(\left.M\right|_{E_{i}}\right)$. Then $\left.M \cong \bigoplus_{i=1}^{t} M\right|_{E_{i}}$. 
[This document contains the author's accepted manuscript. For the publisher's version, see the link in the header of this document.]

Proof. Because of the assumption on ranks, a basis for $M$ must be a disjoint union of bases of the matroids $\left.M\right|_{E_{i}}$.

Proof of Theorem 1.

We first reduce to the case where $n$ is square-free. Let $s=p_{1} \cdots p_{r}$ denote the square-free part of $n$, and let

$$
t=\frac{n}{s}=p_{1}^{m_{1}-1} \cdots p_{r}^{m_{r}-1} .
$$

Note that

$$
\operatorname{rank} \mu_{n}=\phi(n)=t \cdot \phi(s)=t \cdot \operatorname{rank} \mu_{s} .
$$

For $0 \leq j \leq t-1$, let $E_{j}:=\left\{\zeta^{j}, \zeta^{j+t}, \ldots, \zeta^{j+(s-1) t}\right\}$. Then

$$
Z_{n}=\bigsqcup_{j=0}^{t-1} E_{j}
$$

and the submatroid $\left.\mu_{n}\right|_{E_{j}}$ obtained by restricting $\mu_{n}$ to the ground set $E_{j}$ is isomorphic to $\mu_{s}$. By Lemma 3, it follows that $\mu_{n}$ is isomorphic to a direct sum of $t$ copies of $\mu_{s}$. The following figure illustrates the decomposition for $n=18$, $s=6$ and $t=3$.

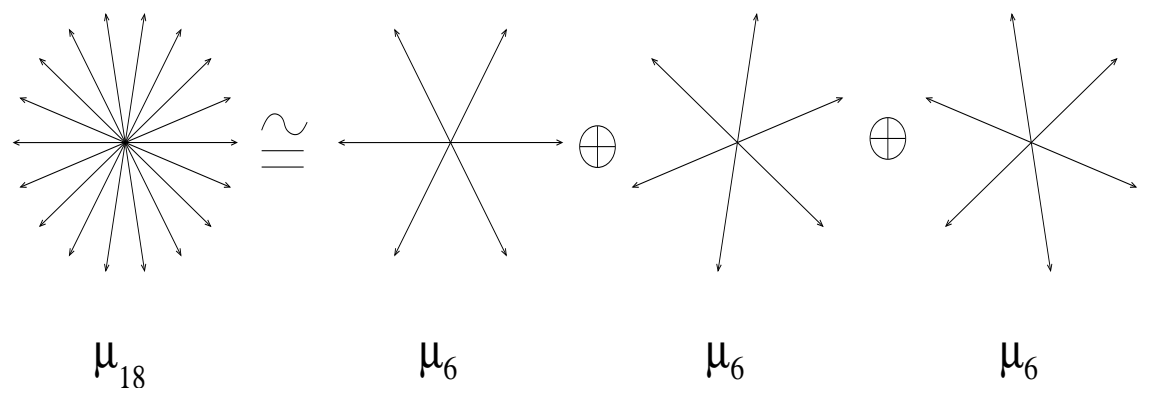

Since duality commutes with direct sums, we have reduced to the case that $n=p_{1} \cdots p_{r}$ is square-free, and assume this for the remainder of the proof.

For each prime $p$ dividing $n$, consider the short exact sequence of $\mathbb{Q}$-vector spaces

$$
0 \rightarrow \mathbb{Q} \stackrel{i_{p}}{\longrightarrow} \mathbb{Q}^{p} \stackrel{\pi_{p}}{\longrightarrow} \mathbb{Q}\left(\zeta_{p}\right) \rightarrow 0
$$

in which $\pi_{p}$ maps the $j^{\text {th }}$ standard basis element $e_{j}$ in $\mathbb{Q}^{p}$ to $\zeta_{p}^{j}$, and the image of $i_{p}$ is the line $\mathbb{Q}\left(e_{1}+\cdots+e_{n}\right)$. Rephrasing this, the cochain complex $\mathcal{C}(p)$ defined by

$$
\begin{aligned}
& 0 \rightarrow \mathcal{C}^{0}(p) \longrightarrow \mathcal{C}^{1}(p) \rightarrow 0 \\
& 0 \rightarrow \mathbb{Q} \stackrel{i_{p}}{\longrightarrow} \mathbb{Q}^{p} \rightarrow 0
\end{aligned}
$$


[This document contains the author's accepted manuscript. For the publisher's version, see the link in the header of this document.]

has

$$
\begin{aligned}
& H^{0}(\mathcal{C}(p))=0, \\
& H^{1}(\mathcal{C}(p))=\mathbb{Q}\left(\zeta_{p}\right)\left(\cong \mathbb{Q}^{p-1}\right) .
\end{aligned}
$$

Note that the complex $\mathcal{C}(p)$ coincides, up to a shift in homological degree, with the augmented simplicial cochain complex that computes the cohomology of the 0 -dimensional complex $\Delta_{p}^{0}$. Similarly, the tensor product of complexes

$$
\mathcal{C}:=\bigotimes_{j=1}^{r} \mathcal{C}\left(p_{j}\right)
$$

coincides with the augmented simplicial cochain complex for the simplicial join $\Delta_{p_{1}, \ldots, p_{r}} \cong \Delta_{p_{1}}^{0} * \cdots * \Delta_{p_{r}}^{0}$. The Künneth formula over $\mathbb{Q}$ then implies that the complex $\mathcal{C}$ has no homology except in the last position. The last cochain group in $\mathcal{C}$ maps surjectively onto the homology as follows:

$$
\mathcal{C}^{r}:=\bigotimes_{j=1}^{r} \mathcal{C}^{1}\left(p_{j}\right) \cong \bigotimes_{j=1}^{r} \mathbb{Q}^{p_{j}} \stackrel{\otimes_{j} \pi_{p_{j}}}{\longrightarrow} \bigotimes_{j=1}^{r} \mathbb{Q}\left(\zeta_{p_{j}}\right)
$$

The Chinese Remainder Theorem allows us to identify the last map in (3) with the surjection $\mathbb{Q}^{n} \stackrel{\pi_{n}}{\rightarrow} \mathbb{Q}(\zeta)$ sending $e_{j}$ to $\zeta^{j}$, that is, the map whose matrix has columns representing $\mu_{n}$. Because $\mathcal{C}$ has no other homology, the last coboundary map $\delta: \mathcal{C}^{r-1} \rightarrow \mathcal{C}^{r}$ has columns spanning the kernel of $\pi_{n}$. Its transpose

$$
C_{r-1}\left(\Delta_{p_{1}, \ldots, p_{r}} ; \mathbb{Q}\right) \stackrel{\delta^{T}}{\longrightarrow} C_{r-2}\left(\Delta_{p_{1}, \ldots, p_{r}} ; \mathbb{Q}\right)
$$

has columns which represent the simplicial matroid $\mathcal{S}\left(\Delta_{p_{1}, \ldots, p_{r}}, \mathbb{Q}\right)$, hence this matroid is dual to $\mu_{n}$.

We close this section with a few remarks.

Remark 4. To give some further context for Theorem 1, it should be noted that matroid duality has played a key role in the study of simplicial matroids from the beginning. Crapo and Rota studied the full simplicial matroid $\mathcal{S}_{m-k}^{m}(\mathbb{F})$ corresponding to the $(k-1)$-skeleton of the full simplex with $m$ vertices, and observed that Alexander duality implies that $\mathcal{S}_{k}^{m}(\mathbb{F})$ and $\mathcal{S}_{m-k}^{m}(\mathbb{F})$ are dual matroids [6, Proposition 11.4]; see also [17, Theorem 6.2.1]. Lindström [11] observed another interesting example of such a duality, this time involving two natural matroids (one of them simplicial), both of whose ground sets are indexed by the bases of some (other) simple matroid; see [17, §6.5].

Remark 5. It has been observed (e.g., by Johnsen [9]) that the primitive $n^{\text {th }}$ roots of unity form a $\mathbb{Q}$-basis for $\mathbb{Q}(\zeta)$ if and only if $n$ is square-free. Thus if $n=p_{1} \cdots p_{r}$ is square-free, one might ask which particular basis of the simplicial matroid $\mathcal{S}\left(\Delta_{p_{1}, \ldots, p_{r}}^{r-1}, \mathbb{Q}\right)$ (that is, which higher-dimensional tree $T$ of $\left.\Delta_{p_{1}, \ldots, p_{r}}^{r-1}\right)$ corresponds to the set of primitive $n^{\text {th }}$ roots. Passing through matroid duality, and the Chinese Remainder Theorem identification in the proof of the theorem, one can check that $T$ is the union of the stars within $\Delta_{p_{1}, \ldots, p_{r}}^{r-1}$ of the vertices 
[This document contains the author's accepted manuscript. For the publisher's version, see the link in the header of this document.]

$v_{1}, \ldots, v_{r}$, where $v_{i}$ is the unique vertex of $\Delta_{p_{i}}^{0}$ labeled by 0 modulo $p_{i}$. Applying a simple nerve argument to this cover of $T$ by stars, one finds that $T$ is not only $\mathbb{Q}$-acyclic as expected, but in fact contractible. Hence it has vanishing homology group $\tilde{H}_{r-2}\left(\Delta_{T}, \mathbb{Z}\right)$, and contributes exactly 1 in the summation of equation (2).

Remark 6. If $n$ is divisible by at most two primes (that is, $r \leq 2$ above), then the complex $\Delta_{p_{1}, p_{2}}^{r-1}$ is a graph. In this case, the matroid $\mu_{n}$ is cographic (see, e.g., [17, p. 35], and Section 3 below).

Furthermore, if $n$ is odd, then the cyclotomic matroid $\mu_{2 n}$ is the parallel extension of $\mu_{n}$ in which one creates one parallel copy for each ground set element. For instance, $\mu_{n}$ is again cographic for $n=2 p q$, where $p, q$ are odd primes: it is the cographic matroid of the graph obtained by duplicating every edge of the complete bipartite graph $K_{p, q}$.

\section{The CASE OF TWO PRIME FACTORS}

The goal of this section is to compute some enumerative invariants of the cyclotomic matroid $\mu_{n}$, finer than the number of bases, in the special case where $n=p_{1}^{m_{1}} p_{2}^{m_{2}}$ has only two prime factors.

The Tutte polynomial $T_{M}(x, y)$ is one of the most important isomorphism invariants of a matroid $M$; see the excellent survey by Brylawski and Oxley [18, Chapter 6] for background. We begin by reviewing briefly the definition of the Tutte polynomial, as well as some elementary properties relevant for our calculations.

Let $M$ be a matroid with ground set $E$, and let $r$ be the rank function on subsets $A$ of $E$. When $M$ is representable, $r(A)$ is the dimension of the linear span of the vectors in $A$. The Tutte polynomial $T_{M}(x, y)$ may be defined as the corank-nullity generating function

$$
T_{M}(x, y):=\sum_{A \subset E}(x-1)^{r(M)-r_{M}(A)}(y-1)^{|A|-r_{M}(A)}
$$

$[18, \S 6.2]$. Two easy consequences of (4) are as follows. First, if $M^{*}$ is the dual matroid to $M$, then

$$
T_{M^{*}}(x, y)=T_{M}(y, x) .
$$

Second, setting $y=1$ and replacing $x$ with $x+1$ in (4) gives a generating function for independent subsets of $M$ according to their cardinality:

$$
T_{M}(x+1,1)=\sum_{\text {independent } I \subset E} x^{r(M)-|I|} .
$$

Let $G=(V, E)$ be a graph, and let $M=M(G)$ be the corresponding graphic matroid on ground set $E$, whose bases are the spanning trees of $G$ and whose independent sets are acyclic subgraphs (= forests). Then there is a substitution 
[This document contains the author's accepted manuscript. For the publisher's version, see the link in the header of this document.]

of variables in $T_{G}:=T_{M(G)}$ which gives Crapo's coboundary polynomial $\bar{\chi}_{G}(q, t)[18, \S 6.3 \mathrm{~F}]:$

$$
\begin{aligned}
\bar{\chi}_{G}(q, t) & :=q^{-1} \sum_{\substack{\text { vertex colorings } \\
f: V \rightarrow\{1,2, \ldots, q\}}} t^{\left|\left\{\left\{v_{1}, v_{2}\right\} \in E: f\left(v_{1}\right)=f\left(v_{2}\right)\right\}\right|} \\
& =(t-1)^{|V|-c(G)} T_{G}\left(\frac{q+t-1}{t-1}, t\right)
\end{aligned}
$$

where $c(G)$ denotes the number of connected components of $G$. Note that $T_{G}(x, y)$ may be recovered from $\bar{\chi}_{G}(x, y)$ via the substitution $q=(x-1)(y-1), t=$ $y$, and that the usual chromatic polynomial $\chi_{G}(q)$ is the specialization $q \bar{\chi}_{G}(q, 0)$.

In this section, we consider the special case that $n=p_{1}^{m_{1}} p_{2}^{m_{2}}$. By Theorem 1, the cyclotomic matroid $\mu_{n}$ is dual to the direct sum of $p_{1}^{m_{1}-1} p_{2}^{m_{2}-1}$ copies of the simplicial matroid $\mathcal{S}\left(\Delta_{p_{1}, p_{2}}^{1} ; \mathbb{Q}\right)$. As mentioned previously, the 1-dimensional complex $\Delta_{p_{1}, p_{2}}^{1}$ is simply the complete bipartite graph $K_{p_{1}, p_{2}}$, and the simplicial matroid is the usual graphic matroid $M\left(K_{p_{1}, p_{2}}\right)$.

We begin by obtaining an exponential generating function for the coboundary polynomials of these cycle matroids, mimicking Ardila's method in [1, Theorem 2.4.1]. Let $K_{p_{1}, p_{2}}$ have bipartite vertex set $V_{1} \sqcup V_{2}$ with $\left|V_{i}\right|=p_{i}$. Every vertexcoloring $f: V \rightarrow\{1,2, \ldots, q\}$ decomposes the partite sets $V_{1}, V_{2}$ into (possibly empty) color classes $V_{1}^{(i)}, V_{2}^{(i)}$ :

$$
V_{1}=\bigsqcup_{i=1}^{q} V_{1}^{(i)}, \quad V_{2}=\bigsqcup_{i=1}^{q} V_{2}^{(i)}
$$

with

$$
\left|\left\{\left\{v_{1}, v_{2}\right\} \in E: f\left(v_{1}\right)=f\left(v_{2}\right)\right\}\right|=\sum_{i=1}^{q}\left|V_{1}^{(i)}\right|\left|V_{2}^{(i)}\right| .
$$

Now exponential generating function manipulation (see [14, Prop. 5.1.3]) gives the following formula.

Proposition 7. Denote by $\bar{\chi}_{p_{1}, p_{2}}(q, t)$ the coboundary polynomial of the graphic matroid of the complete bipartite graph $K_{p_{1}, p_{2}}$. Then

$$
1+q\left(\sum_{\substack{\left(p_{1}, p_{2}\right) \in \\ \mathbb{N}^{2}-\{(0,0)\}}} \bar{\chi}_{p_{1}, p_{2}}(q, t) \frac{x_{1}^{p_{1}} x_{2}^{p_{2}}}{p_{1} ! p_{2} !}\right)=\left(\sum_{\left(m_{1}, m_{2}\right) \in \mathbb{N}^{2}} t^{m_{1} m_{2}} \frac{x_{1}^{m_{1}} x_{2}^{m_{2}}}{m_{1} ! m_{2} !}\right)^{q} .
$$

This formula does not appear to generalize to the case that $n$ has more than two prime factors. 
[This document contains the author's accepted manuscript. For the publisher's version, see the link in the header of this document.]

Remark 8. Setting $t=0$ yields the following simple exponential generating function for the chromatic polynomial $\chi_{p_{1}, p_{2}}(q)$ of $K_{p_{1}, p_{2}}$ :

$$
1+\sum_{\left(p_{1}, p_{2}\right) \in \mathbb{N}^{2}-\{(0,0)\}} \chi_{p_{1}, p_{2}}(q) \frac{x_{1}^{p_{1}} x_{2}^{p_{2}}}{p_{1} ! p_{2} !}=\left(e^{x_{1}}+e^{x_{2}}-1\right)^{q} .
$$

We now apply the generating function for coboundary polynomials to enumerate the $\mathbb{Q}$-linearly independent subsets of the $n^{t h}$ roots of unity. Setting $t=y+1$ and $x_{i}=z_{i} / y$ in the formula of Proposition 7 and applying (7) gives

$$
\begin{array}{r}
y^{-1} \sum_{\left(p_{1}, p_{2}\right) \in \mathbb{N}^{2}-\{(0,0)\}} T_{K_{p_{1}, p_{2}}}\left(\frac{q+y}{y}, y+1\right) \frac{z_{1}^{p_{1}} z_{2}^{p_{2}}}{p_{1} ! p_{2} !}= \\
q^{-1}\left[\left(\sum_{\left(m_{1}, m_{2}\right) \in \mathbb{N}^{2}} \frac{(y+1)^{m_{1} m_{2}}}{y^{m_{1}+m_{2}}} \frac{z_{1}^{m_{1}} z_{2}^{m_{2}}}{m_{1} ! m_{2} !}\right)^{q}-1\right] .
\end{array}
$$

Multiplying through by $y$ and taking the limit as $q$ approaches 0 (via L'Hôpital's Rule), we obtain

$$
\begin{gathered}
\sum_{\left(p_{1}, p_{2}\right) \in \mathbb{N}^{2}-\{(0,0)\}} T_{K_{p_{1}, p_{2}}}(1, y+1) \frac{z_{1}^{p_{1}} z_{2}^{p_{2}}}{p_{1} ! p_{2} !}= \\
z \log \left(\sum_{\left(m_{1}, m_{2}\right) \in \mathbb{N}^{2}} \frac{(y+1)^{m_{1} m_{2}}}{y^{m_{1}+m_{2}}} \frac{z_{1}^{m_{1}} z_{2}^{m_{2}}}{m_{1} ! m_{2} !}\right) .
\end{gathered}
$$

Once again, recall that for $n=p_{1}^{m_{1}} p_{2}^{m_{2}}$, Theorem 1 implies that the cyclotomic matroid $\mu_{n}$ is dual to the direct sum of $p_{1}^{m_{1}-1} p_{2}^{m_{2}-1}$ copies of the graphic matroid $M\left(K_{p_{1}, p_{2}}\right)$. Therefore, (5) and (6) imply the following result.

Corollary 9. Let $n=p_{1}^{m_{1}} p_{2}^{m_{2}}$, where $p_{1}, p_{2}$ are distinct primes.

Then the generating function

$$
\sum_{\substack{I \subset Z_{n} \\ \mathbb{Q}-\text { linearly independent }}} y^{\phi(n)-|I|}
$$

equals the $\left(p_{1}^{m_{1}-1} p_{2}^{m_{2}-1}\right)^{\text {th }}$ power of the coefficient of $\frac{z_{1}^{p_{1}} z_{2}^{p_{2}}}{p_{1} ! p_{2} !}$ in

$$
y \log \left(\sum_{\left(m_{1}, m_{2}\right) \in \mathbb{N}^{2}} \frac{(y+1)^{m_{1} m_{2}}}{y^{m_{1}+m_{2}}} \frac{z_{1}^{m_{1}} z_{2}^{m_{2}}}{m_{1} ! m_{2} !}\right) .
$$

Remark 10. One can deduce from equation (8) the generating function counting acyclic orientations of $K_{p_{1}, p_{2}}$; see [14, Ex. 5.6]. Ardila [2, Prop. 1] has recently generalized this acyclic orientation calculation in a direction different from ours, 
[This document contains the author's accepted manuscript. For the publisher's version, see the link in the header of this document.]

by computing the generating function for faces of various dimensions in the hyperplane arrangement associated to $K_{p_{1}, p_{2}}$.

We conclude this section with an observation about the independent set polynomial $T_{M}(x+1,1)$ (see $\left.(6)\right)$ for $M=M\left(K_{p, q}\right)$. Note that $T_{M}(x+1,1)$ enumerates spanning subsets of $Z_{p q}$ by cardinality when $p, q$ are distinct primes. Computations for small values of $p, q$ suggest that for $p \leq q$ (not necessarily prime), $T_{M\left(K_{p, q}\right)}(x+1,1)$ is divisible by $(x+p)^{q-p+1}$. This follows from a more refined statement (Proposition 11 below) on the enumeration of spanning forests in $K_{p, q}$. Let $K_{p, q}$ have disjoint vertex sets $V=\left\{v_{1}, \ldots, v_{p}\right\}$ and $W=\left\{w_{1}, \ldots, w_{q}\right\}$. For a subset $F$ of its edges and $u$ a vertex, $\operatorname{let}^{\operatorname{deg}_{F}}(u)$ denote the degree of $u$ in the edge-subgraph corresponding to $F$. Define a forest enumerator

$$
A_{p, q}=A_{p, q}\left(x_{1}, \ldots, x_{p}\right):=\sum_{\text {forests } F} x_{1}^{\operatorname{deg}_{F}\left(v_{1}\right)} \ldots x_{p}^{\operatorname{deg}_{F}\left(v_{p}\right)} .
$$

For example, it can be calculated that

$$
\begin{aligned}
& A_{1, q}=\left(1+x_{1}\right)^{q}, \\
& A_{2, q}=\left(1+x_{1}+x_{2}\right)^{q-1}\left(1+x_{1}+x_{2}+q x_{1} x_{2}\right) .
\end{aligned}
$$

Note that $A_{p, q}$ specializes to the independent set polynomial of $K_{p, q}$ as follows. Since $K_{p, q}$ has $p q$ edges, each of which is incident to exactly one $v_{i}$, we obtain

$$
T_{K_{p, q}}(x+1,1)=x^{p q} A_{p, q}\left(x^{-1}, \ldots, x^{-1}\right) .
$$

Proposition 11. Let $q \geq p \geq 1$ be integers. Then

$$
A_{p, q}=\sum_{j=0}^{p-1}\left(1+x_{1}+\cdots+x_{p}\right)^{q-j} \tilde{A}_{p, j}
$$

where $\tilde{A}_{p, q}:=\sum_{F} x_{1}^{\operatorname{deg}_{F}\left(v_{1}\right)} \ldots x_{p}^{\operatorname{deg}_{F}\left(v_{p}\right)}$, in which the summation is over spanning forests $F$ in $K_{p, q}$ in which every vertex $w_{i}$ has degree at least 2.

In particular, $A_{p, q}$ is divisible by

$$
\left(1+x_{1}+\cdots+x_{p}\right)^{q-p+1} .
$$

Proof. Given $F$ a spanning forest of $K_{p, q}$, let $W^{\prime} \subset W$ be the set of $w_{i}$ for which $\operatorname{deg}_{F}\left(w_{j}\right)>1$. We claim that $\left|W^{\prime}\right|<p$, else $F$ contains at least $2 p$ edges among the $2 p$ vertices $\left|W^{\prime} \cup V\right|$, which contradicts the assumption that $F$ is acyclic.

After choosing the restriction of $F$ to the vertices $W^{\prime} \cup V$, the remaining edges of $F$ consist of at most one edge incident to each vertex in $W-W^{\prime}$. Classifying $F$ according to the cardinality $\left|W^{\prime}\right|=j$, the assertion follows.

The quotient $\frac{A_{p, q}}{\left(1+x_{1}+\cdots+x_{p}\right)^{q-p+1}}$ is a symmetric polynomial of total degree $2(p-$ 1 ) in the variables $x_{1}, \ldots, x_{p}$. It appears not to factor further in general. One might also hope for a factorization of a forest enumerator that keeps track of the degrees in $V$ as well as those in $W$, but this also appears not to factor further. 
Cyclotomic and simplicial matroids (with Victor Reiner) Israel J. Math. 150 (2005), 229--240. The original publication is available at www.springerlink.com: http://dx.doi.org/10.1007/BF02762381, Open Access version: http://kuscholarworks.ku.edu/dspace/.

\section{[This document contains the author's accepted manuscript. For the publisher's version, see the link in the header of this document.]}

\section{REFERENCES}

[1] F. Ardila, Enumerative and algebraic aspects of matroids and hyperplane arrangements. Ph.D. thesis, MIT, 2003.

[2] F. Ardila, A tropical morphism related to the hyperplane arrangement of the complete bipartite graph. Preprint, arXiv:math.C0/0404287, 2004.

[3] R.M. Adin, Counting colorful multi-dimensional trees. Combinatorica 12 (1992), 247-260.

[4] L.W. Beineke and R.E. Pippert, Properties and characterizations of $k$-trees. Mathematika 18 (1971), 141-151.

[5] E.D. Bolker, Simplicial geometry and transportation polytopes. Trans. Amer. Math. Soc. 217 (1976), 121-142.

[6] H.H. Crapo and G.-C. Rota, On the foundations of combinatorial theory: Combinatorial geometries. Preliminary edition. The M.I.T. Press, Cambridge, Mass.-London, 1970.

[7] S. Faridi, The facet ideal of a simplicial complex. Manuscripta Math. 109 (2002), no. 2, 159-174.

[8] F. Harary and E.M. Palmer, On acyclic simplicial complexes. Mathematika 15 (1968), $115-122$.

[9] K. Johnsen, Lineare Abhängigkeiten von Einheitswurzeln. Elem. Math. 40 (1985), 57-59.

[10] G. Kalai, Enumeration of $\mathbb{Q}$-acyclic simplicial complexes. Israel J. Math. 45 (1983), 337351.

[11] B. Lindström, Matroids on the bases of simple matroids. Europ. J. Combin. 2 (1981), 61-63.

[12] J.R. Munkres, Elements of algebraic topology. Addison-Wesley Publishing Company, Menlo Park, CA, 1984.

[13] J.G. Oxley, Matroid theory. Oxford Science Publications. The Clarendon Press, Oxford University Press, New York, 1992.

[14] R.P. Stanley, Enumerative Combinatorics, vol. 2. Cambridge University Press, Cambridge, 1999.

[15] D.J.A. Welsh, Matroid theory. London Math. Soc. Monographs 8. Academic Press, London-New York, 1976.

[16] N. White, Theory of matroids. Encyclopedia of Mathematics and its Applications 26. Cambridge University Press, Cambridge, 1986.

[17] N. White, Combinatorial geometries. Encyclopedia of Mathematics and its Applications 29. Cambridge University Press, Cambridge, 1987.

[18] N. White, Matroid applications. Encyclopedia of Mathematics and its Applications 40. Cambridge University Press, Cambridge, 1992.

School of Mathematics, University of Minnesota, Minneapolis, MN 55455

E-mail address: martin@math.umn.edu

School of Mathematics, University of Minnesota, Minneapolis, MN 55455

E-mail address: reiner@math.umn.edu 